American J. of Engineering and Applied Sciences 3 (1): 49-55, 2010

ISSN 1941-7020

(C) 2010 Science Publications

\title{
Fixed-Star Tracking Attitude Control of Spacecraft Using Single-Gimbal Control Moment Gyros
}

\author{
${ }^{1}$ Sangwon Kwon, ${ }^{2}$ Yuki Tani, ${ }^{1}$ Hiroshi Okubo and ${ }^{1}$ Takashi Shimomura \\ ${ }^{1}$ Department of Aerospace Engineering, Osaka Prefecture University, \\ Gakuen-Cho 1-1, Sakai, Osaka, Japan \\ ${ }^{2}$ Department of R and D, Advanced Engineering Services Co., Ltd., Tokyo, Japan
}

\begin{abstract}
Problem statement: A cluster of small-sized Single-Gimbal Control Moment Gyros (SGCMGs) is proposed as an attitude control actuator for high-speed maneuver of small satellites. There exists a singularity problem what is peculiar to the CMG system. Approach: This study presented a simple singularity avoidance steering law using the Singular Value Decomposition (SVD) algorithm. Results: Capability of the present steering method in singularity avoidance was demonstrated with numerical simulations for fixed-star tracking control of a small satellite using four SGCMGs. Conclusion: The proposed steering law utilizes the singular value decomposition to obtain singular vectors and generates the command gimbal rate that keeps the command torque in the direction orthogonal to the singular direction with a maximum gain.
\end{abstract}

Key words: Tracking attitude control, control moment gyro, singularity avoidance, SVD

\section{INTRODUCTION}

Recently, many missions using small satellites have been engaged, because small satellites are easier and faster in development than large satellites and thereby, they can provide increased launch opportunities. In development of small satellites, one of the most severe constraints is limited power, mass, or capacity of various devices. In order to perform high-speed maneuver of small satellites, in this study, we develop an attitude control system using Control Moment Gyros (CMGs). Small-sized CMGs installed to a small satellite can provide enough torque, angular momentum and slew rate while not increasing in power, mass, or volume.

In the past, CMGs have been applied to attitude control of large-sized space structures such as the international space station. However, attitude control with CMGs is also effective to small satellites, especially for high speed or large angle maneuver. However, there exists a singularity problem what is peculiar to CMGs. To solve this problem, some singularity avoidance techniques have been developed in several papers (Bedrossian et al., 1990; Ford and Hall, 2000; Wie et al., 2001). The authors also proposed a simple singularity avoidance logic for a set of four SGCMGs using Singular Value Decomposition (SVD) (Tani et al., 2004; Okubo and Tani, 2005).
In this study, we consider a fixed-star tracking control of a spacecraft using four SGCMGs and propose a method to solve the singularity avoidance problem.

Dynamics of spacecraft with CMGs: We briefly describe the mathematical model of a system of redundant CMGs applied to attitude control of a rigid spacecraft. The Euler's equation of motion of a rigid spacecraft equipped with CMGs is described by:

$\dot{\mathrm{H}}+\omega^{\times} \mathrm{H}=0$

Where:

$\mathrm{H} \in \mathfrak{R}^{3}=$ The total angular momentum vector in the body-fixed frame

$\omega \in \mathfrak{R}^{3}=$ The spacecraft angular velocity vector

The notation $\omega^{\times}$denotes the skew-symmetric matrix defined by:

$\omega^{\times}=\left[\begin{array}{ccc}0 & -\omega_{\mathrm{z}} & \omega_{\mathrm{y}} \\ \omega_{\mathrm{z}} & 0 & -\omega_{\mathrm{x}} \\ -\omega_{\mathrm{y}} & \omega_{\mathrm{x}} & 0\end{array}\right]$

The total angular momentum vector $\mathrm{H}$ can be expressed as follows:

Corresponding Author: Sangwon Kwon, Department of Aerospace Engineering, Osaka Prefecture University, Gakuen-Cho 1-1, Sakai, Osaka, Japan 
$\mathrm{H}=\mathrm{J} \omega+\mathrm{h}$

Where:

$$
\begin{aligned}
\mathrm{J} \in \mathfrak{R}^{3 \times 3}= & \text { The inertia matrix of the spacecraft including } \\
& \text { CMGs }
\end{aligned}
$$

$\mathrm{h} \in \mathfrak{R}^{3}=$ The $\mathrm{CMG}$ angular momentum vector expressed in the body-fixed frame

Substituting Eq. 3 into Eq. 1, we obtain:

$(\mathrm{J} \dot{\omega}+\dot{\mathrm{h}})+\omega^{\times}(\mathrm{J} \omega+\mathrm{h})=0$

Equation 4 can be divided into the following two equations if the internal torque generated by CMGs is denoted as $\tau \in \Re^{3}$ :

$\mathrm{J} \dot{\omega}+\omega^{\times} \mathrm{J} \omega=\tau$

$\dot{\mathrm{h}}+\omega^{\times} \mathrm{h}=-\tau$

Thus the dynamic equation of motion of a spacecraft equipped with CMGs consists of the dynamics of the spacecraft (Eq. 5a) and the dynamics of the CMGs (Eq. 5b). The desired CMG angular momentum rate for generating the spacecraft control torque is given by:

$\dot{\mathrm{h}} \equiv \mathrm{T}=-\tau-\omega^{\times} \mathrm{h}$

The CMG angular momentum vector $h=h(\delta)$ is a function of the gimbal angle vector $\delta \in \Re^{\mathrm{n}}$, then the time derivative of the angular momentum is obtain as:

$\dot{\mathrm{h}}=\frac{\partial \mathrm{h}}{\partial \delta} \frac{\mathrm{d} \delta}{\mathrm{dt}} \equiv \mathrm{G} \dot{\delta}$

Where:

$\mathrm{G}=\mathrm{G}(\delta)=$ The $3 \times \mathrm{n}$ Jacobian matrix

$\mathrm{n}=$ The number of the CMGs

$\dot{\delta} \in \mathfrak{R}^{\mathrm{n}} \quad=$ The gimbal rate command and is obtained as:

$\dot{\delta}=\mathrm{G}^{+} \mathrm{T}$

where, $G^{+}=G^{T}\left(G G^{T}\right)^{-1}$ is the pseudo-inverse of matrix G.

In order to describe the attitude kinematics, we have the following quaternion kinematic differential equation:

$$
\left[\begin{array}{c}
\dot{\mathrm{q}}_{1} \\
\dot{\mathrm{q}}_{2} \\
\dot{\mathrm{q}}_{3} \\
\dot{\mathrm{q}}_{4}
\end{array}\right]=\frac{1}{2}\left[\begin{array}{cccc}
0 & \omega_{\mathrm{z}} & -\omega_{\mathrm{y}} & \omega_{\mathrm{x}} \\
-\omega_{\mathrm{z}} & 0 & \omega_{\mathrm{x}} & \omega_{\mathrm{y}} \\
\omega_{\mathrm{y}} & -\omega_{\mathrm{x}} & 0 & \omega_{\mathrm{z}} \\
-\omega_{\mathrm{x}} & -\omega_{\mathrm{y}} & -\omega_{\mathrm{z}} & 0
\end{array}\right]\left[\begin{array}{l}
\mathrm{q}_{1} \\
\mathrm{q}_{2} \\
\mathrm{q}_{3} \\
\mathrm{q}_{4}
\end{array}\right]
$$

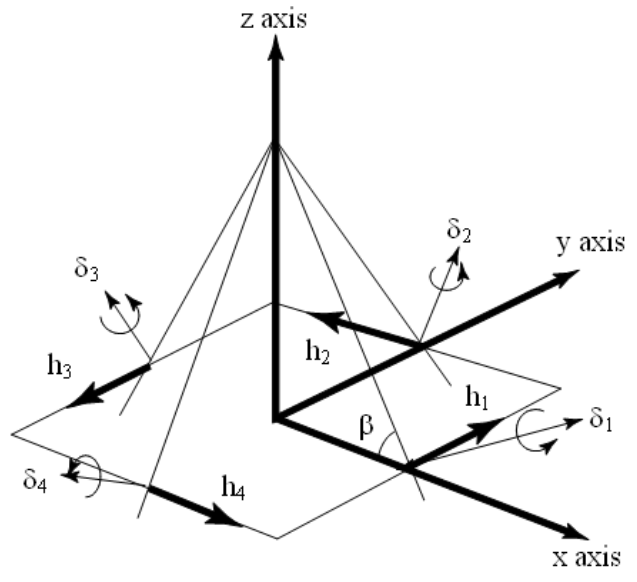

Fig. 1: Pyramid configuration for four SGCMGs

Pyramid array of four SGCMGs: Setting $n=4$, we consider a pyramid array of four SGCMGs as shown in Fig. 1, where four SGCMGs are located on the faces of pyramid and the gimbal axes are orthogonal to the pyramid faces. Each SGCMG has the same angular momentum and the skew angle is chosen as $\beta=54.73$ (deg) so that the momentum envelope becomes nearly spherical. The angular momentum vector $h$ is given as a function of gimbal angle $\delta$ as:

$$
\mathrm{h}=\mathrm{h}_{\mathrm{w}}\left[\begin{array}{c}
-\mathrm{c} \beta \sin \delta_{1}-\cos \delta_{2}+\mathrm{c} \beta \sin \delta_{3}+\cos \delta_{4} \\
\cos \delta_{1}-\mathrm{c} \beta \sin \delta_{2}-\cos \delta_{3}+\mathrm{c} \beta \sin \delta_{4} \\
\mathrm{~s} \beta \sin \delta_{1}+\mathrm{s} \beta \sin \delta_{2}+\mathrm{s} \beta \sin \delta_{3}+\mathrm{s} \beta \sin \delta_{4}
\end{array}\right]
$$

where, $h_{w}$ is the angular momentum of a wheel, $\mathrm{c} \beta=\cos \beta$ and $\mathrm{s} \beta=\sin \beta$ and the Jacobian matrix $\mathrm{G}$ is given by:

$$
\mathrm{G}=\mathrm{h}_{\mathrm{w}}\left[\begin{array}{cccc}
-\mathrm{c} \beta \cos \delta_{1} & \sin \delta_{2} & \mathrm{c} \beta \cos \delta_{3} & -\sin \delta_{4} \\
-\sin \delta_{1} & -\mathrm{c} \beta \cos \delta_{2} & \sin \delta_{3} & \mathrm{c} \beta \cos \delta_{4} \\
\mathrm{~s} \beta \cos \delta_{1} & \mathrm{~s} \beta \cos \delta_{2} & \mathrm{~s} \beta \cos \delta_{3} & \mathrm{~s} \beta \cos \delta_{4}
\end{array}\right]
$$

\section{MATERIALS AND METHDOS}

Singularity avoidance using SVD: A singularity is encountered when there exists some direction for which the array of CMGs is not capable of producing torque. This phenomenon occurs when the gimbal angles of CMGs become some specific arrangement. The $3 \times 4$ Jacobian matrix $\mathrm{G}$ is a function of the gimbal angles, as shown in Eq. 11 and it has the maximum rank of 3. When $\operatorname{rank}(\mathrm{G})=2$, Eq. 11 deteriorates, where all column vectors of Jacobian matrix $G$ become coplanar and there exists a unit vector $\mathrm{u}_{\mathrm{s}}$ orthogonal to that coplanar plane; i.e.: 
$\mathrm{G}^{\mathrm{T}} \mathrm{u}_{\mathrm{s}}=0$

Therefore, the CMG system cannot produce any momentum along the direction of singular vector $\mathrm{u}_{\mathrm{s}}$.

In this study, we propose a method of singularity avoidance that uses the singular value decomposition to obtain the singular direction and output the torque orthogonal to the singular direction for fast singularity avoidance.

First, we consider the singular value decomposition of the Jacobian matrix G. For such G, there exist unitary matrices $U \in \mathfrak{R}^{3 \times 3}$ and $V \in \mathfrak{R}^{4 \times 4}$ such that $\mathrm{U}^{\mathrm{T}} \mathrm{U}=\mathrm{I}_{3}$ and $\mathrm{V}^{\mathrm{T}} \mathrm{V}=\mathrm{I}_{4}$ and:

$\mathrm{G}=\mathrm{U \Sigma} \mathrm{V}^{\mathrm{T}}$

where:

$\Sigma=\left[\begin{array}{cccc}\sigma_{1} & 0 & 0 & 0 \\ 0 & \sigma_{2} & 0 & 0 \\ 0 & 0 & \sigma_{3} & 0\end{array}\right]$

The positive numbers, $\sigma_{1} \geq \sigma_{2} \geq \sigma_{3} \geq 0$, are called singular values of matrix $\mathrm{G}$.

From Eq. 13, for $1 \leq i \leq 3$, we have:

$\left(\mathrm{GG}^{\mathrm{T}}\right) \mathrm{U}=\mathrm{U}\left(\Sigma \Sigma^{\mathrm{T}}\right)$ or $\left(\mathrm{GG}^{\mathrm{T}}\right) \mathrm{u}_{\mathrm{i}}=\sigma_{\mathrm{i}}^{2} \mathrm{u}_{\mathrm{i}}$

$\left(\mathrm{G}^{\mathrm{T}} \mathrm{G}\right) \mathrm{V}=\mathrm{V}\left(\Sigma^{\mathrm{T}} \Sigma\right)$ or $\left(\mathrm{G}^{\mathrm{T}} \mathrm{G}\right) \mathrm{v}_{\mathrm{i}}=\sigma_{\mathrm{i}}^{2} \mathrm{v}_{\mathrm{i}}$

and $\left(\mathrm{G}^{\mathrm{T}} \mathrm{G}\right) \mathrm{v}_{4}=0$, where:

$\mathrm{U}=\left[\begin{array}{lll}\mathrm{u}_{1} & \mathrm{u}_{2} & \mathrm{u}_{3}\end{array}\right]$

$\mathrm{V}=\left[\begin{array}{llll}\mathrm{v}_{1} & \mathrm{v}_{2} & \mathrm{v}_{3} & \mathrm{v}_{4}\end{array}\right]$

The column vector $u_{i}$ and $v_{i}$ are the left and right singular vectors of matrix $G$, respectively. The pseudo inverse of matrix $\mathrm{G}$ can be expanded with Eq. 13 in terms of the singular vectors $\mathrm{u}_{\mathrm{i}}$ and $\mathrm{v}_{\mathrm{i}}$ as follows:

$\mathrm{G}^{+}=\mathrm{V} \Sigma^{+} \mathrm{U}^{\mathrm{T}}$

Where:

$$
\Sigma^{+}=\left[\begin{array}{ccc}
1 / \sigma_{1} & 0 & 0 \\
0 & 1 / \sigma_{2} & 0 \\
0 & 0 & 1 / \sigma_{3} \\
0 & 0 & 0
\end{array}\right]
$$

Therefore, the pseudo-inverse steering law in Eq. 8 can be written as follows:

$$
\dot{\delta}=\mathrm{G}^{+} \mathrm{T}=\sum_{\mathrm{i}=1}^{3}\left(\frac{1}{\sigma_{\mathrm{i}}}\right) \mathrm{v}_{\mathrm{i}} \mathrm{u}_{\mathrm{i}}{ }^{\mathrm{T}} \mathrm{T}
$$

If $\sigma_{i}$ is zero in a singularity, the gimbal rate command diverges to the infinity. The singularity robust steering law (SR steering law) is a method to avoid such a singularity (Wie et al., 2001). In this method, the gimbal rate command is given by the following equation:

$\dot{\delta}=\mathrm{G}^{\#} \mathrm{~T}$

where, $G^{\#}$ is called the SR inverse given by:

$\mathrm{G}^{\#}=\mathrm{G}^{\mathrm{T}}\left(\mathrm{GG}^{\mathrm{T}}+\lambda \mathrm{I}\right)^{-1}$

and $\lambda$ is a constant positive scalar to be properly selected. Note that:

$\mathrm{G}^{\#}=\mathrm{V} \Sigma^{\#} \mathrm{U}^{\mathrm{T}}$

where:

$\Sigma^{\#}=\left[\begin{array}{ccc}\sigma_{1} /\left(\sigma_{1}^{2}+\lambda\right) & 0 & 0 \\ 0 & \sigma_{2} /\left(\sigma_{2}^{2}+\lambda\right) & 0 \\ 0 & 0 & \sigma_{3} /\left(\sigma_{3}^{2}+\lambda\right) \\ 0 & 0 & 0\end{array}\right]$

At a singular point with $\operatorname{rank}(\mathrm{G})=2$ and $\sigma_{3}=0$, vectors $u_{3}$ and $v_{3}$ represent the singular torque and the singular gimbal rate direction, respectively. Then:

$\dot{\delta}=\sum_{\mathrm{i}=1}^{2}\left(\frac{\sigma_{\mathrm{i}}}{\sigma_{\mathrm{i}}^{2}+\lambda}\right) \mathrm{v}_{\mathrm{i}} \mathbf{u}_{\mathrm{i}}^{\mathrm{T}} \mathrm{T}$

Now, we introduce an evaluation function for indicating that the system is approaching to a singularity. The following singularity parameter $\kappa$ is defined as an index of the degree of singularity:

$\kappa \equiv \frac{\sigma_{1}}{\sigma_{3}}$

where, $\sigma_{1}$ and $\sigma_{3}$ are the maximum and the minimum singular value of matrix $G$, respectively. The value of $\kappa$ increases, as the gimbal angles approaches to a singular point with $\sigma_{3}$ being a very small value. In this study, we propose the following steering law: 
$\dot{\delta}=\sum_{\mathrm{i}=1}^{3}\left(\frac{\sigma_{\mathrm{i}}}{\sigma_{\mathrm{i}}^{2}+\lambda}\right) \mathrm{v}_{\mathrm{i}} \mathrm{u}_{\mathrm{i}}^{\mathrm{T}} \mathrm{T}+\mathrm{k}_{\mathrm{SA}} \mathrm{SW}(\kappa) \mathrm{v}_{1}$

where, $\mathrm{SW}(\kappa)$ is a switching function defined as:

$$
\mathrm{SW}(\kappa)=\frac{1}{2}\left(\frac{\kappa-\kappa_{\mathrm{d}}}{\left|\kappa-\kappa_{\mathrm{d}}\right|}+1\right)
$$

The first term in Eq. 26 is the same as the SR steering law in Eq. 20 and the second term is added to escape from the singularities. When $\kappa$ is smaller than $\kappa_{\mathrm{d}}$, the switching function $\mathrm{SW}(\kappa)=0$ and the proposed steering law in Eq. 26 reduces to the conventional SR steering law in Eq. 20.

Fixed star tracking attitude control: We consider fixed-star tracking attitude control of a spacecraft. Suppose that there are two fixed stars in the inertial frame as shown in Fig. 2. The line-of-sight vector of the remote sensor is aligned along $\mathrm{z}$ axis of the body-fixed frame.

A coordinate transformation matrix $\mathrm{C}$ from the inertial frame to the body-fixed frame is defined as:

$$
C=\left[\begin{array}{ccc}
\cos \theta & 0 & -\sin \theta \\
\sin \phi \sin \theta & \cos \phi & \sin \varphi \cos \theta \\
\cos \phi \sin \theta & -\sin \phi & \cos \varphi \cos \theta
\end{array}\right]
$$

where, $\phi$ and $\theta$ are the Euler angle about $\mathrm{x}$ axis and $\mathrm{y}$ axis of the body-fixed frame, respectively. The direction vector of star $i$, for $i=a, b$ in the body-fixed frame is defined as $\mathrm{s}_{\mathrm{i}}{ }^{\mathrm{b}}=\left[\mathrm{s}_{\mathrm{i} 1}{ }^{\mathrm{b}}, \mathrm{s}_{\mathrm{i} 2}{ }^{\mathrm{b}}, \mathrm{s}_{\mathrm{i} 3}{ }^{\mathrm{b}}\right]^{\mathrm{T}}$.
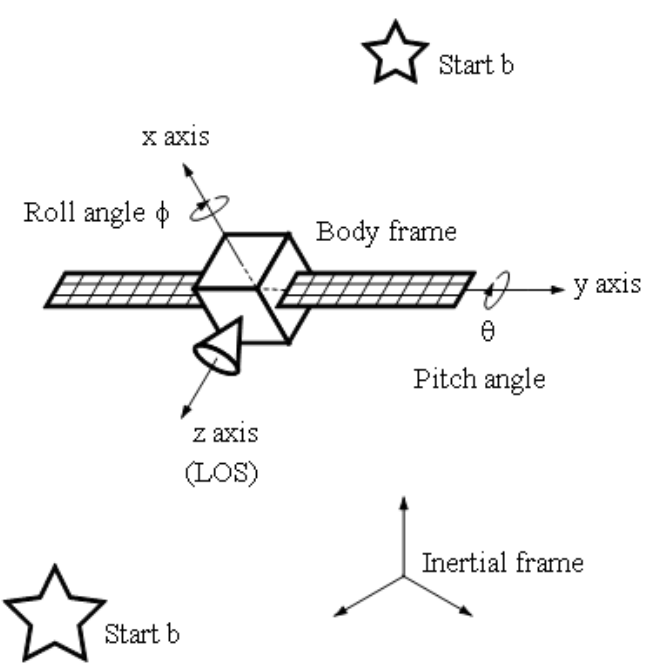

Fig. 2: Configuration of two fixed-stars
Therefore, we obtain:

$\left[\begin{array}{l}\mathrm{s}_{\mathrm{i} 1}{ }^{\mathrm{b}} \\ \mathrm{s}_{\mathrm{i} 2}{ }^{\mathrm{b}} \\ \mathrm{s}_{\mathrm{i} 3}{ }^{\mathrm{b}}\end{array}\right]=\mathrm{C}\left[\begin{array}{l}0 \\ 0 \\ 1\end{array}\right]=\left[\begin{array}{c}-\sin \theta \\ \sin \phi \cos \theta \\ \cos \phi \cos \theta\end{array}\right]$

From Eq. 29, $\theta$ and $\phi$ are calculated as:

$\theta=-\operatorname{asin}\left(s_{i 1}^{b}\right)$

$\phi=\operatorname{atan} 2\left(\frac{\mathrm{s}_{\mathrm{i} 2}{ }^{\mathrm{b}}}{\cos \theta}, \frac{\mathrm{s}_{\mathrm{i} 3}{ }^{\mathrm{b}}}{\cos \theta}\right)$

A PD controller is designed as follows:

$$
\tau=-\mathrm{K}_{\mathrm{p}}\left[\begin{array}{c}
\phi \\
\theta \\
0
\end{array}\right]-\mathrm{K}_{\mathrm{d}}\left[\begin{array}{c}
\omega_{\mathrm{x}} \\
\omega_{\mathrm{y}} \\
\omega_{\mathrm{z}}
\end{array}\right]
$$

where $\mathrm{K}_{\mathrm{p}} \in \mathfrak{R}^{3 \times 3}, \mathrm{~K}_{\mathrm{d}} \in \mathfrak{R}^{3 \times 3}$ are the gain matrices.

\section{RESULTS AND DISCUSSION}

Numerical simulation: We show a numerical example of the tracking control for two fixed stars, star a and star $b$. The spacecraft parameters, the initial condition and the control gain are given in Table 1. The direction vectors of two fixed stars in the inertial frame are given as:

$\mathrm{s}_{\mathrm{a}}=[0,0,1]^{\mathrm{T}}, \mathrm{s}_{\mathrm{b}}=[0,0,-1]^{\mathrm{T}}$

In this study, the constant positive scalar $\lambda$ in (Wie et al., 2001) is chosen as:

$$
\lambda=0.01 \exp \left(-10 \operatorname{det}\left(\mathrm{GG}^{\mathrm{T}}\right)\right)
$$

Table 1: Numerical simulation data

\begin{tabular}{lll}
\hline Symbol & Value & Units \\
\hline $\mathrm{J}$ & $\operatorname{diag}[10,9,8]$ & $\mathrm{Kgm}^{2}$ \\
$\mathrm{~h}_{\mathrm{w}}$ & 0.5 & $\mathrm{Nms}$ \\
$\omega_{0}$ & {$[0,0,0]^{\mathrm{T}}$} & $\mathrm{deg} \mathrm{sec}$ \\
$\delta_{0}$ & {$[0,0,0,0]^{\mathrm{T}}$} & $\mathrm{deg}$ \\
$\dot{\delta}_{0}$ & {$[0,0,0,0]^{\mathrm{T}}$} & $\mathrm{rad} \mathrm{sec}^{-1}$ \\
$\left.\dot{\delta}\right|_{\max }$ & 1 & $\mathrm{rad} \mathrm{sec}^{-1}$ \\
$\beta$ & 54.73 & $\mathrm{deg}$ \\
$\mathrm{K}_{\mathrm{SA}}$ & 0.01 & - \\
$\mathrm{K}_{\mathrm{d}}$ & 4.0 & - \\
$\mathrm{K}_{\mathrm{p}}$ & $\operatorname{diag}[5,4.5,4]$ & - \\
$\mathrm{K}_{\mathrm{d}}$ & $\operatorname{diag}[20,18,16]$ & - \\
\hline
\end{tabular}




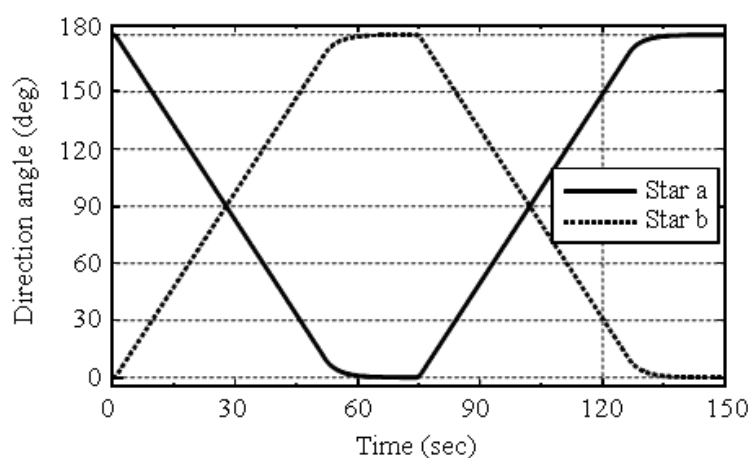

(a)

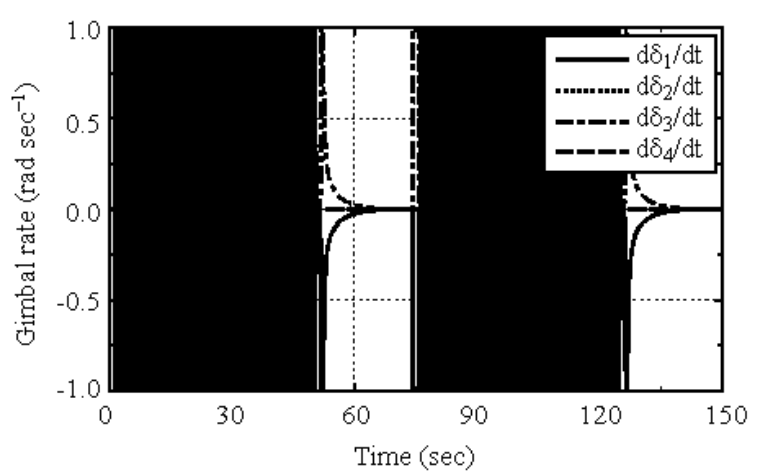

(c)

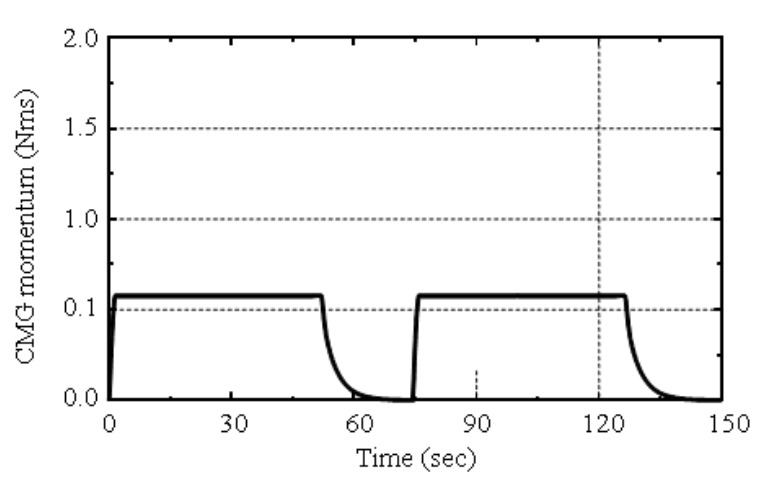

(e)

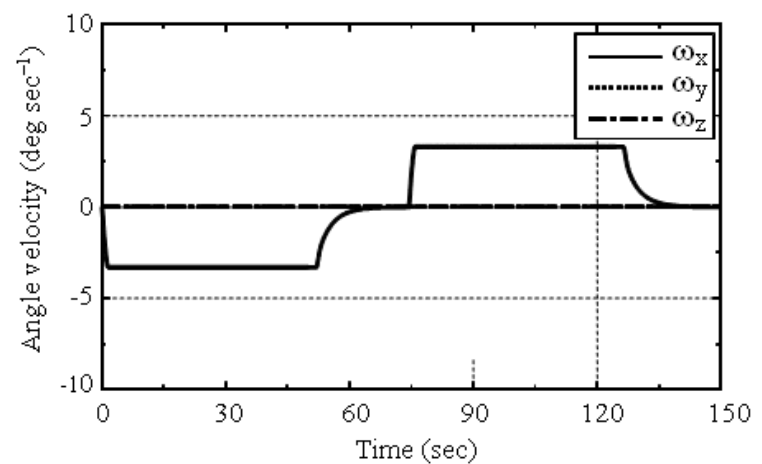

(b)

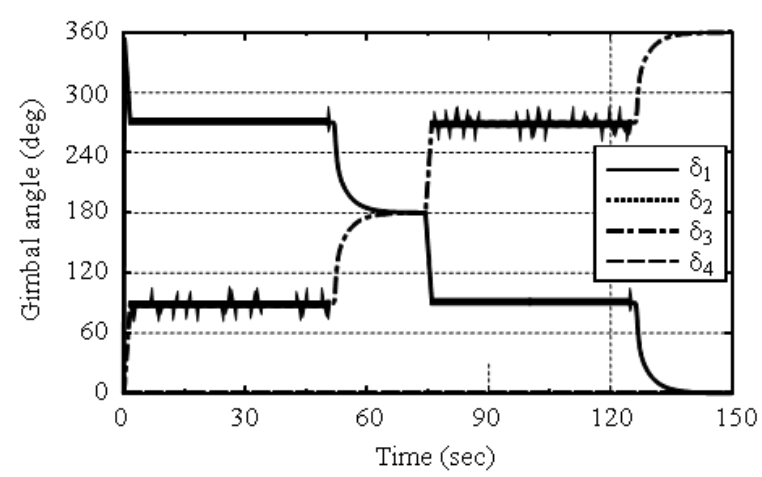

(d)

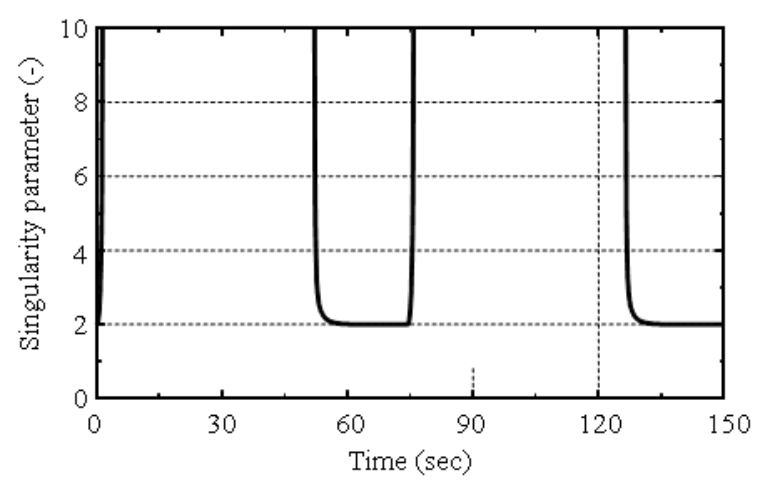

(f)

Fig. 3: Simulation result: SR steering law

A simulation result of the tracking control with the conventional SR steering law in Eq. 20 is shown in Fig. 3 and that with the proposed steering law in Eq. 26 is shown in Fig. 4. In Fig. 3 and 4, (a) shows the direction angle; (b) the spacecraft angular velocity; (c) the gimbal rate; (d) the gimbal angle; (e) the angular momentum of SGCMGs and (f) the singularity parameter $\kappa$. In Fig. $3 a$ and $4 a$, the direction angle is given as the angle between $\mathrm{z}$ axis (LOS) of the body-fixed frame and the line connecting two fixed stars, star a and star $\mathrm{b}$ in the inertial frame.

In Fig. 3 associated with the SR steering law in Eq. 20, the tracking control of star $\mathrm{a}$ and $\mathrm{b}$ is completed in about $150 \mathrm{sec}$. The CMG system encounters the internal singularity of $\delta=[270,0,90,0]^{\mathrm{T}} \mathrm{deg}$ at about $2 \mathrm{sec}$. The fixed star a is tracked in about $75 \mathrm{sec}$.

Similarly, the singularity of $\delta=[90,0,270,0]^{\mathrm{T}} \mathrm{deg}$ is encountered at about $77 \mathrm{sec}$. The fixed star b is tracked in about $150 \mathrm{sec}$. 
Am. J. Engg. \& Applied Sci., 3 (1): 49-55, 2010

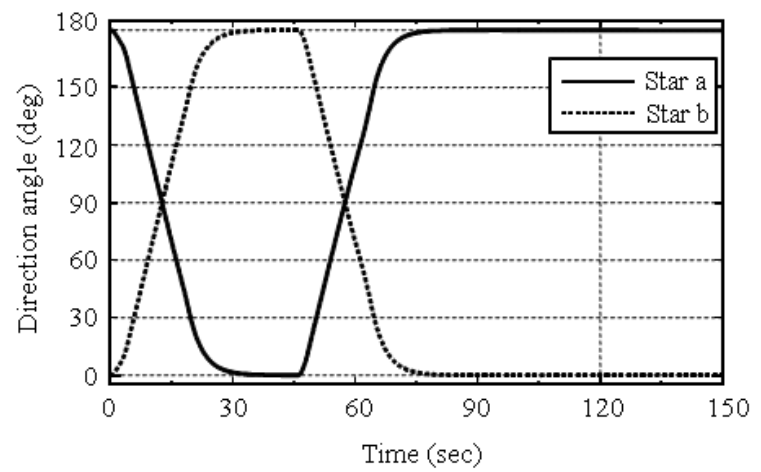

(a)

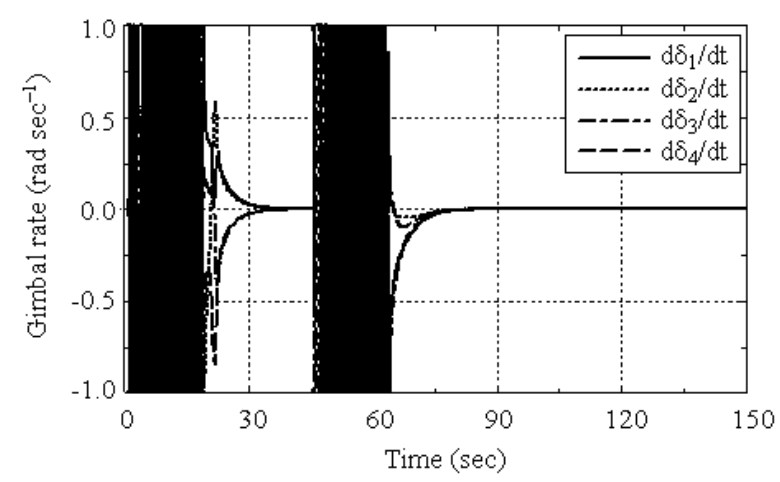

(c)

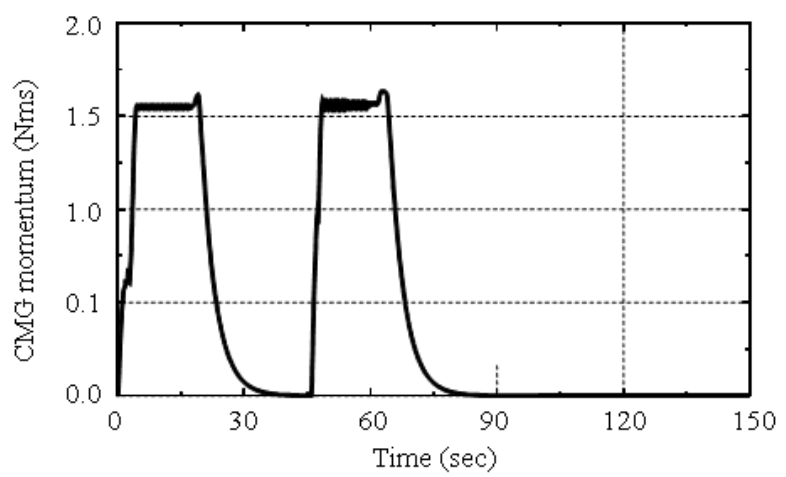

(e)

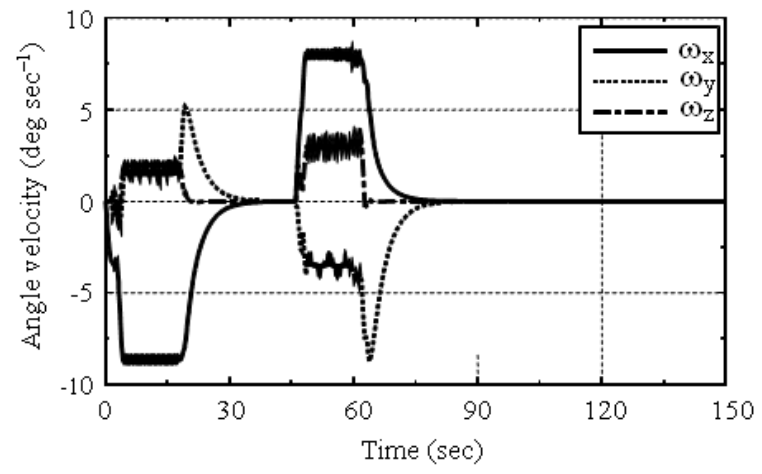

(b)

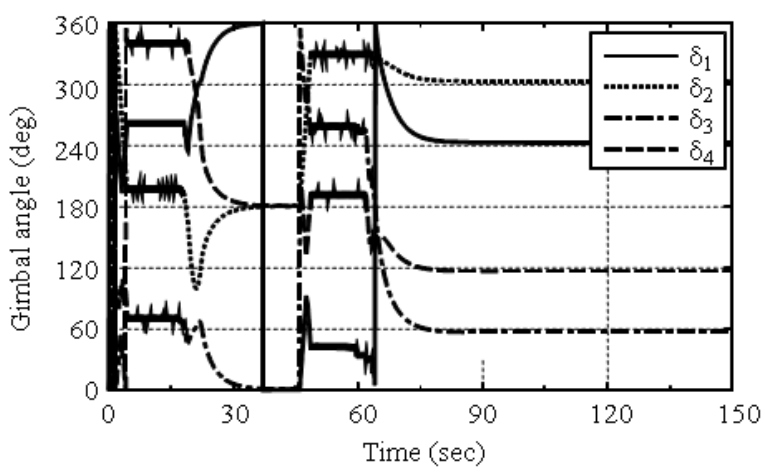

(d)

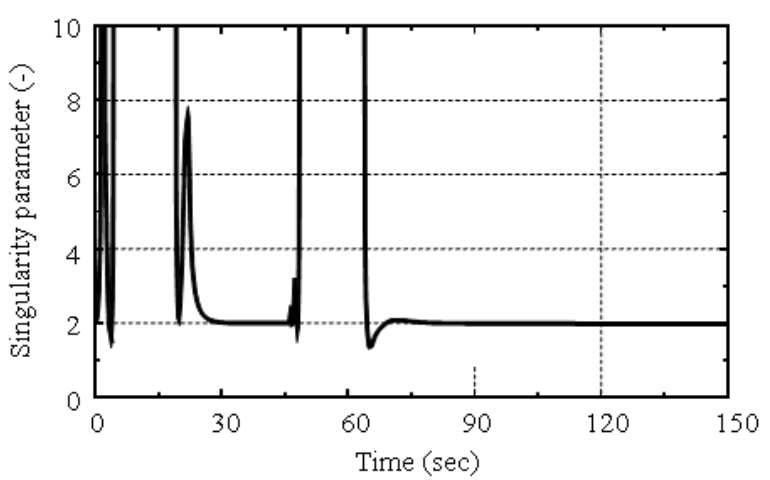

(f)

Fig. 4: Simulation result: proposed steering law

In Fig. 4 associated with the proposed steering law in Eq. 26, a singularity is also encountered at about $2 \mathrm{sec}$, where the CMG system is avoiding the singularity by the output torque in the direction perpendicular to the singular direction. For this reason, the maximum output angular momentum of the CMG system is about $1.56 \mathrm{Nms}$ in the case of the proposed steering law in Eq. 26, whereas it is only about $0.56 \mathrm{Nms}$ when the SR steering law in Eq. 20 is applied.
The result of the numerical simulation demonstrates the advantage of the proposed method in singularity avoidance over the conventional SR steering law. The SR algorithm simply utilizes an artificially perturbed command torque in order to avoid the singularity, whereas the present method efficiently generates the command torque in the direction orthogonal to the singular direction with a maximum gain to escape from the singular point rapidly. 


\section{CONCLUSION}

In this study, a cluster of four SGCMGs in pyramid configuration has been studied for fixed-star tracking control of a small satellite. A new and simple steering law for singularity avoidance has been proposed. The proposed method utilizes the SVD to obtain the singular vector and generates the command gimbal rate that keeps the command torque in the direction orthogonal to the singular direction with a maximum gain. A numerical example of the fixed-star tracking control has been demonstrated to show the advantage of the proposed method over the conventional SR steering law.

\section{REFERENCES}

Bedrossian, N.S., J. Paradiso, E.V. Bergmann and D. Rowell, 1990. Steering law design for redundant singlegimbal control moment gyroscopes. J. Guid. Control Dyn., 13: 1083-1089. http://adsabs.harvard.edu/abs/1990JGCD...13.1083 $\mathrm{B}$

Ford, K.A. and C.D. Hall, 2000. Singular direction avoidance steering for control moment gyros. J. Guid. Control Dyn., 23: 648-656. http://www.aoe.vt.edu/ cdhall/papers/fordhalljgcd. pdf

Okubo, H. and Y. Tani, 2005. Singularity robust steering of redundant single gimbal control moment gyros for small satellites. In: Proceeding of the 8th International Symposium on Artificial Intelligence, Robotics and Automation in Space, Sept. 5-8, Munich, Germany, pp: 1-8.
Tani, Y., H. Okubo, H. Tokutake and H. Azuma, 2004. Singularity robust control of redundant control moment gyros for small satellites. Proceeding of the 24th International Symposium on Space Technology and Science, 30 May-6 June, JSASS, Miyazaki, Japan, pp: 1-6.

Wie, B., D. Bailey and C. Heiberg, 2001. Singularity robust steering logic for redundant single gimbal control moment gyros. J. Guid. Control Dyn., 24: 865-872.

http://direct.bl.uk/bld/PlaceOrder.do?UIN=101943

938\&ETOC $=$ RN\&from=searchengine 